\title{
Parasutterella excrementihominis gen. nov., sp. nov., a member of the family Alcaligenaceae isolated from human faeces
}

Correspondence

Fumiko Nagai

fumiko-nagai@yakult.co.jp

\author{
Fumiko Nagai, Masami Morotomi, Hiroshi Sakon and Ryuichiro Tanaka \\ Yakult Central Institute for Microbiological Research, 1796 Yaho, Kunitachi, 186-8650 Tokyo, \\ Japan
}

\begin{abstract}
A novel, strictly anaerobic, non-spore-forming, Gram-negative coccobacillus (strain YIT $11859^{\top}$ ) was isolated from human faeces. Biochemically, this strain was largely unreactive and was asaccharolytic. Growth of strain YIT $11859^{\top}$ in peptone-yeast extract broth produced no visible turbidity, and a trace amount of propionate was detected as an end product of metabolism. 16S rRNA gene sequence analysis showed that strain YIT $11859^{\top}$ was related most closely to the type strains of Sutterella species, with $90.8-88.0 \%$ sequence similarity. Phylogenetic analysis of these and other related sequences confirmed that strain YIT $11859^{\top}$ was phylogenetically most closely associated with Sutterella species, but formed a separate cluster, indicating that strain YIT $11859^{\top}$ represents a novel member of the family Alcaligenaceae. Fatty acid analysis demonstrated the presence of a high concentration of $C_{18: 1} \omega 9 c$ ( $75 \%$ of the total). The main respiratory quinones were menaquinone (MK-6) and methylated menaquinone (MMK-6). The $G+C$ content of the DNA was $49.8 \mathrm{~mol} \%$. These results suggest that strain YIT $11859^{\top}$ represents a novel species of a new genus, for which the name Parasutterella excrementihominis gen. nov., sp. nov. is proposed. The type strain of Parasutterella excrementihominis is YIT $11859^{\top}$ $\left(=\mathrm{DSM} 21040^{\top}=\mathrm{JCM} 15078^{\top}\right)$.
\end{abstract}

By using culture-independent approaches based on smallsubunit (16S and 18S) rRNA gene sequence diversity, most bacterial phylotypes identified from the human intestinal microbiota have been assigned to seven phyla, of which members of the Firmicutes and Bacteroidetes are dominant (Rajilić-Stojanović et al., 2007; Eckburg et al., 2005; Hayashi et al., 2002; Wang et al., 2003). The proteobacteria are usually secondary to the above, and members of the classes Alphaproteobacteria, Betaproteobacteria, Gammaproteobacteria, Deltaproteobacteria and Epsilonproteobacteria have been identified (Eckburg et al., 2005). With conventional culture techniques, on the other hand, species of the Proteobacteria such as Escherichia coli, Klebsiella pneumoniae, Enterobacter cloacae and some species of Proteus and Citrobacter have been identified from the human intestinal microbiota (Finegold et al., 1974; Holdeman et al., 1976; Moore \& Holdeman, 1974), and

Abbreviations: FAME, fatty acid methyl ester; ML, maximum likelihood; $\mathrm{MP}$, maximum parsimony.

The GenBank/EMBL/DDBJ accession number for the 16S rRNA gene sequence of strain YIT $11859^{\top}$ is AB370250.

Maximum-likelihood and maximum-parsimony phylogenetic trees based on $16 S$ rRNA gene sequences and a table giving the cellular fatty acid compositions of strain YIT $11859^{\top}$ and the type strains of related type species in the family Alcaligenaceae are available as supplementary material with the online version of this paper. all of these species belong to the family Enterobacteriaceae, class Gammaproteobacteria, except for Sutterella parvirubra (Sakon et al., 2008) and Sutterella wadsworthensis (Wexler et al., 1996; Engberg et al., 2000), which belong to the family Alcaligenaceae, class Betaproteobacteria. This observation can be attributed, at least to some extent, to the lack of appropriate cultivation techniques.

To understand better the physiological characteristics and function of the majority of the human gastrointestinal microbiota, we have performed several intensive cultivation trials aimed at isolating so-called 'unculturable' or 'asyet-uncultured' bacteria from the human gastrointestinal tract (Sakon et al., 2008; Morotomi et al., 2008).

Here we report the isolation of a novel member of the family Alcaligenaceae isolated from human faeces. Although we propose novel taxonomic units (genus and species) based on a single isolate, this isolate displayed more than $98 \% 16 \mathrm{~S}$ rRNA gene sequence similarity to some of the human intestinal uncultured clones reported by several groups in the USA and other countries, as described below, indicating that these bacteria are common members of the human intestinal microbiota.

A faecal sample was collected from a healthy Japanese male ( 29 years old) and immediately transferred anaerobically. The sample was weighed and diluted with pre-reduced 
$0.1 \mathrm{M}$ PBS (pH 7) in an anaerobic glove box (Coy Laboratory Products), which contained $88 \%$ nitrogen, $7 \%$ hydrogen and $5 \%$ carbon dioxide. Each dilution was then spread on anaerobe basal agar (Oxoid). The medium was adjusted to $\mathrm{pH} 5.0,6.0,7.0,8.0$ or 9.0 with $\mathrm{HCl}$ or $\mathrm{NaOH}$ before use. Plates were incubated at $37{ }^{\circ} \mathrm{C}$ for 3 days in the same anaerobic cabinet. Strain YIT $11859^{\mathrm{T}}$ was isolated from a pH 6.0 plate inoculated with a $10^{-6}$ serially diluted faecal sample. Single colonies were picked and streaked out until single cultures were obtained on the same plate (anaerobe basal agar, $\mathrm{pH}$ 6.0). The end products of bacterial metabolism of glucose, lactate or succinate in pre-reduced peptone-yeast extract (PY) medium (Holdeman et al., 1977) supplemented with glucose, lactate or succinate, respectively, were analysed by HPLC according to a previously described procedure (Chonan et al., 1995). Cellular morphology was recorded after Gramstaining of 3-day-old plates or 1-day broth cultures. Cell dimensions were determined by phase-contrast microscopy (Olympus system microscope BX-50) by using an ocular micrometer $(\times 10)$ and objective lens $(\times 100 / 1.25$ numerical aperture). Biochemical characteristics were determined by using the API Rapid ID 32A, API ZYM and API 20A systems (bioMérieux) according to the manufacturer's instructions. Sensitivity to bile was determined by comparing growth of the strain on modified Gifu anaerobic medium (GAM) agar (Nissui Pharmaceutical) with and without $2 \%$ Bacto oxgall (Difco) after 5 days of incubation. The composition of the modified GAM agar was as given previously (Sakon et al., 2008).

Isoprenoid quinones were extracted as described by Komagata \& Suzuki (1987) and were analysed by using an HPLC-atmospheric pressure chemical ionization-MS/ MS system (API3200; Applied Biosystems) with an Lcolumn ODS $(2.1 \times 150 \mathrm{~mm}$; Chemicals Evaluation and Research Institute) according to a modification of the method of Katsuta et al. (2005). Fatty acid methyl esters (FAMEs) were obtained from lyophilized cells grown on modified GAM agar by saponification, methylation and extraction with minor modifications (Kuykendall et al., 1988) of the method of Miller (1982). FAMEs were identified with a Shimadzu model GC-14A gas chromatograph and a Shimadzu model C-R5A chromatograph-data processor. FAME peaks were identified by using retention time comparisons against standard compounds (bacterial acid methyl ester mix; Supelco).

The DNA G + C content was determined by hydrolysing the DNA enzymically and quantifying the nucleosides by HPLC according to the method of Ezaki et al. (1990).

$16 \mathrm{~S}$ rRNA gene sequences of reference species were retrieved from the DNA DataBank of Japan (DDBJ) by using the program FASTA (Pearson \& Lipman, 1985). The sequences were aligned and used to produce an unrooted phylogenetic tree according to the neighbour-joining method (Saitou \& Nei, 1987) by using CLUSTAL $\mathrm{x}$ (version 1.83) (Thompson et al., 1997). Stability of the groupings was estimated by bootstrap analysis (1000 replications) in CLUSTAL X. All phylogenetic trees were visualized by using the program TreeView version 1.6.6 (Page, 1996). Maximum-parsimony (MP) and maximum-likelihood (ML) methods were used to confirm the phylogenetic placement of the aligned sequences. MP analysis was performed by using the software package MEGA4 (Tamura et al., 2007). The ML tree was constructed via the program PHYML (Guindon \& Gascuel, 2003) by using Kimura's two-parameter nucleotide substitution model (Kimura, 1980). The input file was prepared via the program SEQBOOT within the PHYLIP package (Felsenstein, 2004). 16S rRNA gene sequence similarity values between strain YIT $11859^{\mathrm{T}}$ and all other described species within the family Alcaligenaceae were calculated by using EzTaxon (Chun et al., 2007).

Cells of strain YIT $11859^{\mathrm{T}}$ were Gram-negative, obligately anaerobic, non-motile coccobacilli $(0.4-1.1 \times 0.9-2.6 \mu \mathrm{m})$, occurring singly and in pairs. Colonies after 3 days anaerobic incubation on anaerobe basal agar were translucent, entire, circular, convex and pinpoint. Growth of strain YIT $11859^{\mathrm{T}}$ in PY broth produced no visible turbidity; a trace amount of propionate was detected as an end product of metabolism. Addition of glucose, succinate or lactate did not enhance growth or the production of short-chain fatty acids. The strain was asaccharolytic in API test systems. In the API Rapid ID 32A and API ZYM test systems, strain YIT $11859^{\mathrm{T}}$ was largely unreactive. It was positive for esterase $(\mathrm{C} 4)$, arginine arylamidase and leucine arylamidase and weakly positive for acid phosphatase, alanine arylamidase, alkaline phosphatase, esterase lipase (C8), glycine arylamidase and naphthol-AS-BI-phosphohydrolase. All other test results were negative.

The quinone system of strain YIT $11859^{\mathrm{T}}$ consisted of menaquinone (MK-6) and methylated menaquinone (MMK-6).

The only fatty acid comprising more than $10 \%$ of the total for strain YIT $11859^{\mathrm{T}}$ was $\mathrm{C}_{18: 1} \omega 9 c(74.96 \%)$. Other fatty acids detected were $\mathrm{C}_{14: 0}(6.34 \%), \mathrm{C}_{16: 0} \quad(6.31 \%)$, $\mathrm{C}_{18: 1} \omega 9 t(2.74 \%), \mathrm{C}_{18: 0}(1.99 \%)$ and unknown fatty acids (equivalent chain-lengths of 15.306 and 17.478) $(7.67 \%)$. The detailed cellular fatty acid profiles of strain YIT $11859^{\mathrm{T}}$ and related type species in the family Alcaligenaceae are presented in Supplementary Table S1 (available in IJSEM Online). The cellular fatty acid profile of strain YIT $11859^{\mathrm{T}}$ was clearly different from those of related type species in the family Alcaligenaceae.

An approximately 1500-bp region of the 16S rRNA gene of strain YIT $11859^{\mathrm{T}}$ was sequenced. Database searches of recognized taxa revealed that strain YIT $11859^{\mathrm{T}}$ was related most closely to the type strains of Sutterella species (Sutterella parvirubra, 90.8\% 16S rRNA gene sequence similarity; Sutterella wadsworthensis, $89.3 \%$; Sutterella stercoricanis, $88.0 \%$ ). Levels of $16 \mathrm{~S}$ rRNA gene sequence similarity between strain YIT $11859^{\mathrm{T}}$ and the type strains of all other recognized species within the family Alcaligenaceae were less than $90 \%$, namely species of 
Achromobacter (89.1-88.2\%), Advenella (88.8-84.9\%), Alcaligenes (88.4-86.7\%), Azohydromonas (88.2-87.7\%), Bordetella (89.0-88.5\%), Brackiella (87.6\%), Castellaniella (88.5-88.2\%), Derxia (89.0\%), Kerstersia (88.6\%), Oligella (87.4-86.7\%), Pelistega (87.6\%), Pigmentiphaga (88.8-88.7\%), Pusillimonas (88.3\%), Taylorella (88.5$87.4 \%)$ and Tetrathiobacter (88.4-87.7\%).

Phylogenetic analysis of these and other related sequences of recognized taxa was performed and confirmed that strain YIT $11859^{\mathrm{T}}$ was phylogenetically related most closely to Sutterella species, but formed a separate cluster in the neighbour-joining tree (Fig. 1). Other taxa (e.g. Alcaligenes, Achromobacter or Bordetella species) were more distantly related. These results were consistent with those obtained by using the MP and ML methods (Supplementary Figs S1 and S2). Strain YIT $11859^{\mathrm{T}}$ did, however, show higher levels of $16 \mathrm{~S}$ rRNA gene sequence similarity (99.7-98.2\%) to uncultured human faecal or colonic bacteria (Eckburg et al., 2005; Hold et al., 2002; Mai et al., 2006; Ley et al., 2006) (Fig. 1 and Supplementary Figs S1 and S2).

Table 1 shows the main characteristics of strain YIT $11859^{\mathrm{T}}$ that can be used to differentiate it from members of the genus Sutterella. The G+C content of the DNA of strain YIT $11859^{\mathrm{T}}$ was $49.8 \mathrm{~mol} \%$, whereas that of recognized Sutterella species ranges from 60 to $64.5 \mathrm{~mol} \%$ (Sakon et al., 2008; Greetham et al., 2004).

Although there is no direct evidence that members of the genus represented by strain YIT $11859^{\mathrm{T}}$ (Parasutterella gen. nov.) have been isolated from human faeces as common members of the human indigenous microbiota, evidence in favour of this comes from the fact that many uncultured human intestinal bacterial clones with highly similar $16 \mathrm{~S}$ rRNA gene sequences to that of strain YIT $11859^{\mathrm{T}}$ have been deposited with the DDBJ (Fig. 1).
It is evident from the results presented that strain YIT $11859^{\mathrm{T}}$ recovered from human faeces represents a novel species of a new genus within the family Alcaligenaceae, for which the name Parasutterella excrementihominis gen. nov., sp. nov. is proposed.

\section{Description of Parasutterella gen. nov.}

Parasutterella (Pa.ra.sut.te.rel'la. Gr. prep. para besides, next to; N.L. fem. n. Sutterella name of a bacterial genus; N.L. fem. n. Parasutterella a genus similar to Sutterella).

Cells are Gram-negative, non-motile, strictly anaerobic, non-spore-forming cocci to coccobacilli. Oxidase-, ureaseand catalase-negative. Asaccharolytic. Grow in the presence of bile. Aesculin and gelatin are not hydrolysed and nitrate is not reduced. Indole is not produced. The main respiratory quinones are MK-6 and MMK- 6 . The only major fatty acid ( $>10 \%$ of the total) is $\mathrm{C}_{18: 1} \omega 9 \mathrm{c}$. The type species is Parasutterella excrementihominis.

\section{Description of Parasutterella excrementihominis sp. nov.}

Parasutterella excrementihominis (ex.cre.men.ti.ho'mi.nis. L. n. excrementum excrement; L. n. homo -inis human being, man; N.L. gen. n. excrementihominis of faeces of a human).

The description of the species is as given for the genus above. In addition, cells are approximately $0.4-1.1 \times 0.9-$ $2.6 \mu \mathrm{m}$ in size. After 3 days anaerobic incubation on anaerobe basal agar ( $\mathrm{pH}$ 6.0) colonies are translucent, entire, circular, convex and pinpoint. In the API test systems, positive for esterase (C4), arginine arylamidase and leucine arylamidase, weakly positive for acid phosphatase, alanine arylamidase, alkaline phosphatase, esterase

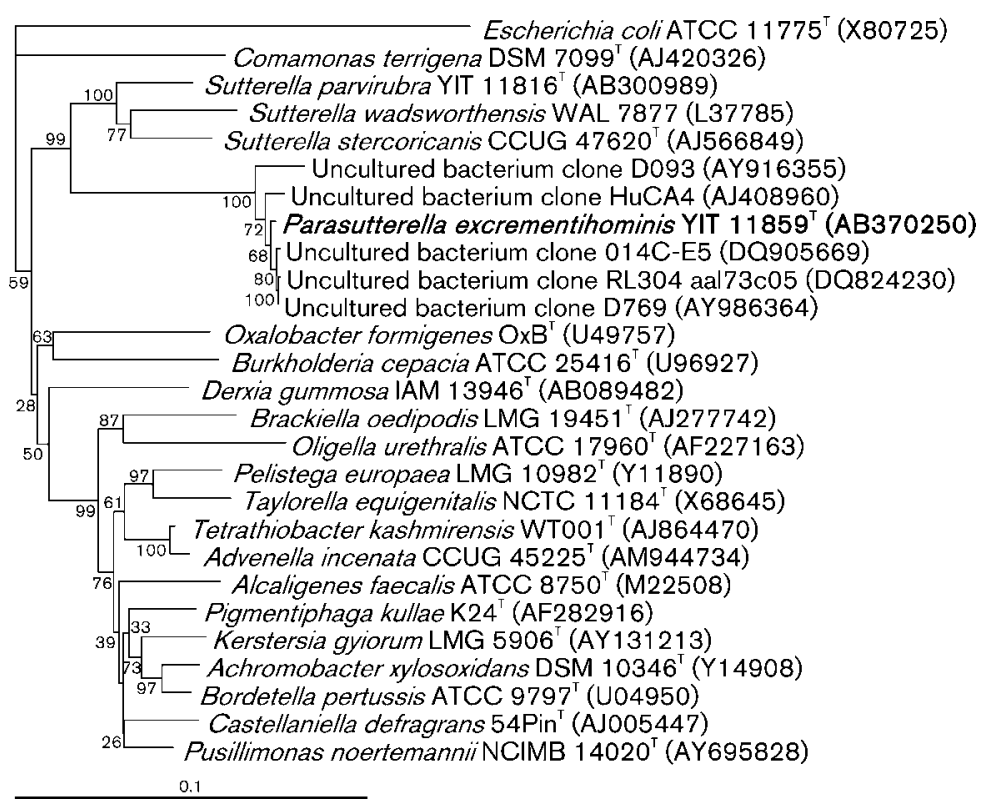

Fig. 1. Phylogenetic tree showing the positions of strain YIT $11859^{\top}$ and the type strains of species of the family A/caligenaceae based on 16S rRNA gene sequence similarity data. The tree was rooted with Escherichia coli ATCC $11775^{\top}$ and was constructed by using the neighbour-joining method, with bootstrap values expressed as percentages of 1000 replications. GenBank/EMBL/DDBJ accession numbers are shown in parentheses. Bar, 0.1 substitutions per nucleotide position. MP and $\mathrm{ML}$ phylogenetic trees are available as Supplementary Figs S1 and S2. 
Table 1. Differential characteristics between strain YIT $11859^{\top}$ and members of the genus Sutterella

Strains: 1, YIT $11859^{\mathrm{T}}$; 2, S. parvirubra YIT $11816^{\mathrm{T}}$; 3, S. wadsworthensis WAL $9799^{\mathrm{T}}$; 4, S. stercoricanis CCUG $47620^{\mathrm{T}}$. Data for strain YIT $11859^{\mathrm{T}}$ and cellular fatty acid data for S. parvirubra YIT $11816^{\mathrm{T}}$ are from the present study. Other data for S. parvirubra YIT $11816^{\mathrm{T}}$, S. wadsworthensis WAL $9799^{\mathrm{T}}$ and S. stercoricanis CCUG $47620^{\mathrm{T}}$ are from Sakon et al. (2008), Wexler et al. (1996) and Greetham et al. (2004), respectively. No data are available for major quinones of Sutterella species. +, Positive; -, negative; w, weakly positive; NA, no data available.

\begin{tabular}{|c|c|c|c|c|}
\hline Characteristic & 1 & 2 & 3 & 4 \\
\hline Isolation source & Human faeces & Human faeces & Gastrointestinal tract infection & Dog faeces \\
\hline Cell morphology & Coccobacilli & Coccobacilli & Straight rods & Short, straight rods \\
\hline Cell size $(\mu \mathrm{m})$ & $0.4-1.1 \times 0.9-2.6$ & $0.4-1 \times 0.4-2$ & $0.5-1 \times 1-3$ & $0.8 \times 1.5-2$ \\
\hline DNA G $+C$ content $(\mathrm{mol} \%)$ & 49.8 & 64.4 & NA & 60 \\
\hline Nitrate reduction & - & - & + & + \\
\hline \multicolumn{5}{|l|}{ API ZYM results } \\
\hline Acid phosphatase & $\mathrm{w}$ & - & NA & - \\
\hline Esterase (C4) & + & - & - & - \\
\hline Esterase lipase (C8) & $\mathrm{w}$ & $\mathrm{w}$ & - & - \\
\hline \multicolumn{5}{|l|}{ Rapid ID 32A API results } \\
\hline Alkaline phosphatase & $\mathrm{w}$ & - & NA & - \\
\hline Arginine arylamidase & + & $\mathrm{w}$ & NA & - \\
\hline Glycine arylamidase & $\mathrm{w}$ & - & NA & $\mathrm{w}$ \\
\hline Leucine arylamidase & + & - & NA & - \\
\hline Major cellular fatty acid(s) & $\mathrm{C}_{18: 1} \omega 9 c$ & $\mathrm{C}_{16: 0}, \mathrm{C}_{18: 1} \omega 9 c$ & $\mathrm{C}_{16: 0}, \mathrm{C}_{16: 1} \omega 7 c, \mathrm{SF} 10^{*}$ & $\mathrm{C}_{16: 0}, \mathrm{C}_{16: 1} \omega 7 c, \mathrm{C}_{18: 1} \omega 9 c$ \\
\hline
\end{tabular}

${ }^{\star}$ Summed feature 10 (SF10) comprises one or more of $\mathrm{C}_{18: 1} \omega 7 c / \omega 9 t / \omega 12 t$ and an unknown peak at 17.834 .

lipase (C8), glycine arylamidase and naphthol-AS-BIphosphohydrolase, but negative for $N$-acetyl- $\beta$-glucosaminidase, $\alpha$-arabinosidase, arginine dihydrolase, chymotrypsin, cystine arylamidase, $\alpha$-fucosidase, $\alpha$-galactosidase, $\beta$ galactosidase, 6 -phospho- $\beta$-galactosidase, $\alpha$-glucosidase, $\beta$ glucosidase, $\beta$-glucuronidase, glutamic acid decarboxylase, glutamyl glutamic acid arylamidase, histidine arylamidase, leucyl glycine arylamidase, lipase (C4), $\alpha$-mannosidase, phenylalanine arylamidase, proline arylamidase, pyroglutamic acid arylamidase, serine arylamidase, trypsin, tyrosine arylamidase and valine arylamidase. The fatty acid profile of the type strain contains $\mathrm{C}_{14: 0}, \mathrm{C}_{16: 0}, \mathrm{C}_{18: 1} \omega 9 c$, $\mathrm{C}_{18: 1} \omega 9 t, \mathrm{C}_{18: 0}$ and unknown fatty acids (equivalent chain-lengths of 15.306 and 17.478). The DNA G+C content of the type strain is $49.8 \mathrm{~mol} \%$.

The type strain, YIT $11859^{\mathrm{T}}\left(=\mathrm{DSM} 21040^{\mathrm{T}}=\mathrm{JCM}\right.$ $\left.15078^{\mathrm{T}}\right)$, was isolated from human faeces.

\section{Acknowledgements}

We are grateful to Dr Jean P. Euzéby of the Ecole Nationale Vétérinaire in Toulouse for his suggestions regarding the etymology of the species epithet. We thank Dr Haruji Sawada for his understanding and encouragement through our research activities.

\section{References}

Chonan, O., Matsumoto, K. \& Watanuki, M. (1995). Effect of galactooligosaccharides on calcium absorption and preventing bone loss in ovariectomized rats. Biosci Biotechnol Biochem 59, 236-239.
Chun, J., Lee, J.-H., Jung, Y., Kim, M., Kim, S., Kim, B. K. \& Lim, Y.-W. (2007). EzTaxon: a web-based tool for the identification of prokaryotes based on $16 \mathrm{~S}$ ribosomal RNA gene sequences. Int J Syst Evol Microbiol 57, 2259-2261.

Eckburg, P. B., Bik, E. M., Bernstein, C. N., Purdom, E., Dethlefsen, L., Sargent, M., Gill, S. R., Nelson, K. E. \& Relman, D. A. (2005). Diversity of the human intestinal microbial flora. Science 308, 1635-1638.

Engberg, J., On, S. L., Harrington, C. S. \& Gerner-Smidt, P. (2000). Prevalence of Campylobacter, Arcobacter, Helicobacter, and Sutterella spp. in human fecal samples as estimated by a reevaluation of isolation methods for campylobacters. J Clin Microbiol 38, 286-291.

Ezaki, T., Saidi, S. M., Liu, S. L., Hashimoto, Y., Yamamoto, H. \& Yabuuchi, E. (1990). Rapid procedure to determine the DNA base composition from small amounts of gram-positive bacteria. FEMS Microbiol Lett 55, 127-130.

Felsenstein, J. (2004). PHYLIP (phylogeny inference package), version 3.6. Distributed by the author. Department of Genome Sciences, University of Washington, Seattle, USA.

Finegold, S. M., Attebery, H. R. \& Sutter, V. L. (1974). Effect of diet on human fecal flora: comparison of Japanese and American diets. Am J Clin Nutr 27, 1456-1469.

Greetham, H. L., Collins, M. D., Gibson, G. R., Giffard, C., Falsen, E. \& Lawson, P. A. (2004). Sutterella stercoricanis sp. nov., isolated from canine faeces. Int J Syst Evol Microbiol 54, 1581-1584.

Guindon, S. \& Gascuel, O. (2003). A simple, fast, and accurate algorithm to estimate large phylogenies by maximum likelihood. Syst Biol 52, 696-704.

Hayashi, H., Sakamoto, M. \& Benno, Y. (2002). Phylogenetic analysis of the human gut microbiota using $16 \mathrm{~S}$ rDNA clone libraries and strictly anaerobic culture-based methods. Microbiol Immunol 46, 535-548.

Hold, G. L., Pryde, S. E., Russell, V. J., Furrie, E. \& Flint, H. J. (2002). Assessment of microbial diversity in human colonic samples by $16 \mathrm{~S}$ rDNA sequence analysis. FEMS Microbiol Ecol 39, 33-39. 
Holdeman, L. V., Good, I. J. \& Moore, W. E. (1976). Human fecal flora: variation in bacterial composition within individuals and a possible effect of emotional stress. Appl Environ Microbiol 31, 359-375.

Holdeman, L. V., Cato, E. P. \& Moore, W. E. C. (1977). Anaerobe Laboratory Manual, 4th edn. Blacksburg, VA: Virginia Polytechnic Institute and State University.

Katsuta, A., Adachi, K., Matsuda, S., Shizuri, Y. \& Kasai, K. (2005). Ferrimonas marina sp. nov. Int J Syst Evol Microbiol 55, 1851-1855.

Kimura, M. (1980). A simple method for estimating evolutionary rates of base substitutions through comparative studies of nucleotide sequences. J Mol Evol 16, 111-120.

Komagata, K. \& Suzuki, K. (1987). Lipid and cell-wall analysis in bacterial systematics. Methods Microbiol 19, 161-207.

Kuykendall, L. D., Roy, M. A., O’Neill, J. J. \& Devine, T. E. (1988). Fatty acids, antibiotic resistance, and deoxyribonucleic acid homology groups of Bradyrhizobium japonicum. Int J Syst Bacteriol 38, 358-361.

Ley, R. E., Turnbaugh, P. J., Klein, S. \& Gordon, J. I. (2006). Microbial ecology: human gut microbes associated with obesity. Nature $\mathbf{4 4 4}$ $1022-1023$.

Mai, V., Greenwald, B., Morris, J. G., Jr, Raufman, J. P. \& Stine, O. C. (2006). Effect of bowel preparation and colonoscopy on postprocedure intestinal microbiota composition. Gut 55, 1822-1823.

Miller, L. T. (1982). Single derivatization method for routine analysis of bacterial whole-cell fatty acid methyl esters, including hydroxy acids. J Clin Microbiol 16, 584-586.

Moore, W. E. \& Holdeman, L. V. (1974). Human fecal flora: the normal flora of 20 Japanese-Hawaiians. Appl Microbiol 27, 961-979.

Morotomi, M., Nagai, F., Sakon, H. \& Tanaka, R. (2008). Dialister succinatiphilus sp. nov. and Barnesiella intestinihominis sp. nov., isolated from human faeces. Int J Syst Evol Microbiol 58, 2716-2720.
Page, R. D. M. (1996). TreeView: an application to display phylogenetic trees on personal computers. Comput Appl Biosci 12, 357-358.

Pearson, W. R. \& Lipman, D. J. (1985). Rapid and sensitive protein similarity searches. Science 227, 1435-1441.

Rajilić-Stojanović, M., Smidt, H. \& de Vos, W. M. (2007). Diversity of the human gastrointestinal tract microbiota revisited. Environ Microbiol 9, 2125-2136.

Saitou, N. \& Nei, M. (1987). The neighbor-joining method: a new method for reconstructing phylogenetic trees. Mol Biol Evol 4, 406425.

Sakon, H., Nagai, F., Morotomi, M. \& Tanaka, R. (2008). Sutterella parvirubra sp. nov. and Megamonas funiformis sp. nov., isolated from human faeces. Int J Syst Evol Microbiol 58, 970-975.

Tamura, K., Dudley, J., Nei, M. \& Kumar, S. (2007). MEGA4: molecular evolutionary genetic analysis (MEGA) software version 4.0. Mol Biol Evol 24, 1596-1599.

Thompson, J. D., Gibson, T. J., Plewniak, F., Jeanmougin, F. \& Higgins, D. G. (1997). The CLUSTAL_X windows interface: flexible strategies for multiple sequence alignment aided by quality analysis tools. Nucleic Acids Res 25, 4876-4882.

Wang, X., Heazlewood, S. P., Krause, D. O. \& Florin, T. H. (2003). Molecular characterization of the microbial species that colonize human ileal and colonic mucosa by using $16 \mathrm{~S}$ rDNA sequence analysis. J Appl Microbiol 95, 508-520.

Wexler, H. M., Reeves, D., Summanen, P. H., Molitoris, E., McTeague, M., Duncan, J., Wilson, K. H. \& Finegold, S. M. (1996). Sutterella wadsworthensis gen. nov., sp. nov., bile-resistant microaerophilic Campylobacter gracilis-like clinical isolates. Int $J$ Syst Bacteriol 46, 252-258. 